\title{
First report of Passiflora edulis symptomless virus in pomegranate in Spain
}

\author{
Celia Canales $^{1} \cdot$ Felix Morán $^{1} \cdot$ Thierry Candresse $^{2} \cdot$ Antonio Olmos $^{1} \cdot$ Ana Belen Ruiz-García ${ }^{1}$ (D)
}

Received: 14 August 2021 / Accepted: 26 August 2021 / Published online: 7 September 2021

(c) Società Italiana di Patologia Vegetale (S.I.Pa.V.) 2021

Keywords PeSV $\cdot$ pomegranate $\cdot$ HTS

Passiflora edulis symptomless virus (PeSV), family Potyviridae, genus Roymovirus, was first identified in Israel infecting Passiflora edulis plants (Jover-Gil et al. 2018). PeSV has also been reported in Turkey, China and India infecting pomegranate (Caglayan et al. 2020). Although it has not been formally associated with symptoms in pomegranate, there are indications that PeSV may induce symptoms in this crop. In June 2020 leaves from a pomegranate growing in Alicante (Spain) and showing chlorotic spots along the veins were analyzed by RNAseq high throughput sequencing (HTS, TrueSeq Illumina technology, 150 bp pairedend). Data was analyzed using CLC Genomics Workbench 10.1.1. After quality control and host genome subtraction, de novo assembly of $1,887,133$ reads generated 10,946 contigs (> $200 \mathrm{nt}$ ), from which 128 were related to PeSV according to BLAST analysis (e-value $<10^{-4}$ ). These contigs showed a high molecular variability indicating a mixed infection by several PeSV isolates. Contig extension performed by Geneious Prime 2020 allowed to recover a 9926 nt near full-length PeSV genomic sequence, (MZ361583, average coverage 528x). This sequence showed 78.1-80.8\% nucleotide identity when compared to PeSV genomic sequences (MT680930-MT680935). No other virus was detected in the HTS analysis. RT-PCR from the original pomegranate plant using the newly designed primers SPeSV-6F (5'-GGCTAG AAACGGTGGGATGA-3') and SPeSV-6R (5'-ACCACC TGGCTCATGGCGA-3') generated the expected $165 \mathrm{nt}$ amplicon, as confirmed by Sanger sequencing $(100 \% \mathrm{nt}$

Ana Belen Ruiz-García

ana.belen.ruiz@uv.es

1 Instituto Valenciano de Investigaciones Agrarias, 46113 Moncada, Valencia, Spain

2 UMR Biologie du Fruit Et Pathologie, Université de Bordeaux, INRAE, 71 Avenue Edouard Bourlaux, 33140 Villenave d'Ornon, France identity with the HTS sequence). In further assays, the 165 nt fragment was also amplified from 17 symptomatic out of 65 tested trees from the same orchard. Although PeSV was not detected in asymptomatic plants, symptomatic trees that tested negative for PeSV were also found. These results could be explained by a limited inclusiveness of the primers used in this study and question the pathogenicity of PeSV in pomegranate. To our knowledge this is the first report of PeSV in Spain.

The authors have no conflicts of interest to declare that are relevant to the content of this article. HTS data supporting the results reported in the article are available from the corresponding author on reasonable request.

\section{References}

Caglayan K, Gazel M, Roumi V, Kocabag HD, Tunç B, Reynard JS, Ruiz-García AB, Olmos A, Candresse T (2020) Identification of Pomegranate as a New Host of Passiflora Edulis Symptomless Virus (PeSV) and Analysis of PeSV Diversity. Agronomy 10:1821. https://doi.org/10.3390/agronomy10111821

Jover-Gil S, Beeri A, Fresnillo P, Samach A, Candela H (2018) Complete genome sequence of a novel virus, classifiable within the Potyviridae family, which infects passion fruit (Passiflora edulis). Arch Virol 163:3191-3194. https://doi.org/10.1007/ s00705-018-3983-7

Publisher's Note Springer Nature remains neutral with regard to jurisdictional claims in published maps and institutional affiliations. 